\title{
Estudo de viabilidade econômica da implantação de uma usina de reciclagem de resíduos da construção civil no município de Pato Branco (PR), utilizando a metodologia multi-índice ampliada
}

\author{
Economic feasibility study of the implementation of a \\ waste recycling plant construction in Pato Branco city, Paraná, \\ using the multi-index methodology extended \\ Luis Carlos Pais Gularte', José Donizetti de Lima², \\ Gilson Adamczuk Oliveira ${ }^{3}$, Marcelo Gonçalves Trentin ${ }^{3}$, Dalmarino Setti ${ }^{3}$
}

\begin{abstract}
RESUMO
Este artigo analisou a viabilidade econômica da instalação de uma usina de reciclagem de resíduos da construção civil, por meio das expectativas de retorno e risco associadas à sua implantação. A análise do investimento em estudo foi realizada utilizando-se a metodologia multi-índice por meio do sistema Web \$LV€P. Também foi realizada uma análise estocástica utilizando a Simulação de Monte Carlo como forma de melhor analisar os riscos envolvidos no projeto. Os resultados mostraram que há viabilidade econômica e baixo risco na implantação dessa usina, pois a probabilidade de o valor presente líquido (VPL) ser menor que zero é de 0,22\%. A análise preliminar realizada mostrou que a decisão de investir nesse empreendimento se apresenta como uma boa opção de investimento, sendo recomendada a sua implementação por ser um projeto com possibilidades de bom retorno financeiro.

Palavras-chave: usina de reciclagem de resíduos da construção civil; viabilidade econômico-financeira; metodologia multi-índice ampliada; \$LV€P; simulação de Monte Carlo.
\end{abstract}

\begin{abstract}
This paper analyzed the economic feasibility of installing a construction waste recycling plant, through the return expectations and risks associated with its implementation. The investment analysis study was performed using multi-index methodology via the web system \$LV€P. A stochastic analysis also was performed using the Monte Carlo simulation, in order to further analyze the risks involved in the project. The results showed that there is low risk and economic feasibility in implementation of this plant, because the probability of the Net Present Value NPV to be zero is less than $0.22 \%$. The performed preliminary analysis showed that the decision to invest in this project is presented as a good investment option, and its implementation is recommended because it is a project with the possibility of a good financial return.
\end{abstract}

Keywords: waste construction recycling plant; economic and financial viability; multi-index methodology extended; \$LV€P; Monte Carlo simulation.

representam cerca de $57 \%$ e são oriundos de demolições, reformas e novas construções. Quando depositados de forma inadequada, geram problemas à sociedade e ao meio ambiente (ABRELPE, 2014).

Com a publicação da Resolução do Conselho Nacional do Meio Ambiente (CONAMA) no 307/02 e da norma técnica NBR 15114/04, da Associação Brasileira de Normas Técnicas (ABNT), foram definidas diretrizes para o gerenciamento dos RCC. A partir dessas normatizações, a responsabilidade pela gestão dos RCC passou da administração pública para os geradores. E, considerando que essa resolução

\section{${ }^{1}$ Conforme destaca Nunes (2004), as terminologias Resíduos Sólidos de Construção e Demolição (RCD) e Resíduos Sólidos da Construção Civil (RCC) vêm sendo difundidas nos meios acadêmicos brasileiros para a denominação de Resíduos da Construção Civil. A Resolução CONAMA n 307/2002 emprega a terminologia Resíduos da Construção Civil, gerando a sigla RCC, a qual será utilizada nesse trabalho. \\ INTRODUÇÃO \\ Com o desenvolvimento das cidades e o rapido crescimento da popula- correto dos resíduos. Dentre os resíduos sólidos urbanos geradosnas cidades brasileiras, os Resíduos da Construção Civil e Demolição (RCC) ${ }^{1}$}

'Mestrando do Programa de Pós-Graduação em Engenharia de Produção e Sistemas pela Universidade Tecnológica Federal do Paraná (UTFPR) - Pato Branco (PR), Brasil. Economista, Técnico Administrativo em Educação na Universidade Federal da Fronteira Sul (UFFS) - Realeza (PR), Brasil.

²Docente do Programa de Pós-Graduação em Engenharia de Produção e Sistemas pela UTFPR - Pato Branco (PR), Brasil. Doutor em Engenharia de Produção pela Universidade Federal do Rio Grande do Sul (UFRGS) - Porto Alegre (RS), Brasil.

${ }^{3}$ Docente do Programa de Pós-Graduação em Engenharia de Produção e Sistemas pela UTFPR - Pato Branco (PR), Brasil. Doutor em Engenharia de Produção pela UFRGS -

Porto Alegre (RS), Brasil.

Endereço para correspondência: Luis Carlos Pais Gularte - Avenida Bruno Zuttion, 2.027 - Centro - 85770-000 - Realeza (PR), Brasil - E-mail: luisgularte755@hotmail.com Recebido: 07/O4/16 - Aceito: 09/09/16 - Reg. ABES: 162097 
preconiza a não geração de resíduos como o elemento básico para a gestão dos canteiros de obras, colocou-se, a partir daí, uma nova realidade para a construção civil no Brasil.

Porém, atualmente, a maioria dos RCC ainda são enviados para aterros, reutilizados ou incinerados, de maneira precária. De acordo com a Associação Brasileira de Empresas de Limpeza Pública e Resíduos Especiais (ABRELPE, 2014), em torno de $42 \%$ de todo o resíduo gerado pelo setor é despejado em aterros. Desse montante, $53 \%$ são reutilizados ou incinerados, o que mostra que apenas $5 \%$ são reciclados. A quantidade de resíduos reciclados no Brasil é baixa se comparada com outros países, como os Estados Unidos, onde se reciclam 140 milhões de toneladas por ano; isso é um reflexo das poucas usinas de reciclagem que atualmente estão instaladas no Brasil (apenas 48), segundo dados da Associação Brasileira para Reciclagem de Resíduos da Construção e Demolição (ABRECON, 2015), sendo que no estado do Paraná há apenas sete em funcionamento.

Azevedo et al. (2006) afirmam que a geração de RCC pode ocorrer nas diferentes fases do ciclo de vida dos empreendimentos: construção, manutenção, reformas e demolição. De acordo com dados da ABRELPE (2014), a geração estimada de RCC no Brasil seria em torno de 122.262 toneladas por dia. Esses dados mostram o elevado potencial que o setor tem em produzir agregados reciclados, os quais podem agregar valor à produção. Com isso, há diminuição da necessidade de matérias-primas virgens, provocando redução nos custos das empresas e também causando impacto socioambiental positivo, uma vez que esses resíduos deixam de ser descartados em aterros ou até no próprio meio ambiente (PINTO, 1999).

Considerando tal cenário, é visível a necessidade de se estudar esse tema. Dessa forma, o objetivo do presente artigo é analisar a viabilidade econômica da implantação de uma usina de reciclagem de resíduos da construção civil e demolição (URRCC) privada para a destinação correta dos RCC no município de Pato Branco (PR). O artigo está dividido da seguinte forma: na seção 1 , foi realizada a introdução do artigo; na seção 2, será feita uma revisão da literatura; a seção 3 apresentará a metodologia; a seção 4, os principais resultados encontrados; por fim, na seção 5 , serão feitas as considerações finais.

\section{REVISÃO DE LITERATURA}

\section{Resíduos da Construção Civil}

A reciclagem de RCC é uma prática que apresenta vantagens ambientais à sociedade, tais como: economia na aquisição de matérias-primas; decréscimo da poluição gerada pelo entulho e de suas consequências, como enchentes e assoreamento de rios e córregos; preservação e redução das áreas de aterros inertes, entre outros (PINTO, 1999). Porém, conforme destacam Santos e Pompeu (2014), para o meio empresarial, o argumento que se refere somente a questões ambientais ou sustentáveis se torna insuficiente, principalmente na atividade proposta, pois a instalação de uma URRCC demanda investimentos elevados. Assim, torna-se necessário, acima de tudo, um planejamento econômico-financeiro para avaliar a viabilidade de um empreendimento desse porte. É necessário justificar a reciclagem desses resíduos, como uma atividade que, entre outros aspectos, gere emprego e renda, e que seja lucrativa economicamente. Os autores ainda destacam que as URRCC produzemzagregado reciclado com variada composição de material que pode ser reaplicado em argamassas e concretos não estruturais, em blocos de concretos e outros produtos.

Analisando especificamente a questão da avaliação do investimento de um projeto desse porte, há muitos estudos que buscaram analisar a viabilidade econômica de se implantar uma URRCC - como exemplos tem-se: Duran et al. (2006), Coelho e Brito (2013), em níveis internacionais, Pinto (1999) e Miranda et al. (2009), em nível nacional. Outros autores analisaram a gestão dessas usinas de reciclagem: Scremin et al. (2014) e Kartam et al. (2004). Porém, conforme destacam Coelho e Brito (2013), a viabilidade econômica de investimento nesse tipo de projeto é uma variável altamente regional, dependente de muitos fatores físicos, econômicos e sociais do local em que ele será inserido. Considerando o forte aspecto regional, o presente estudo, que propõe analisar a viabilidade da implantação de uma URRCC na cidade de Pato Branco (PR), é justificável porque estará incluindo as especificidades do local de instalação na análise.

Devido a esse fator locacional, diferentes resultados têm sido encontrados na literatura: Nunes et al. (2007) não encontraram viabilidade no investimento privado em projetos de novas usinas para os municípios no Brasil. Por outro lado, Zhao et al. (2010) encontraram viabilidade condicional (colocaram a necessidade da intervenção do setor público como fator necessário à viabilidade ou até sugeriram a própria gestão desse) no município de Chongqing, China. Já estudos como o de Duran et al. (2006) apresentaram retornos elevados de investimentos para os municípios irlandeses de Dublin e Limerick, e para uma usina móvel.

Um estudo relevante no que tange à viabilidade econômica da instalação privada de uma URRCC é o de Zhao et al. (2010). Os autores buscaram avaliar se a reciclagem dos RCC seria viável na cidade de Chongqing, China. Ao analisar as condições econômicas da região, eles verificaram que havia uma elevada quantidade de resíduos e uma expressiva demanda por materiais reciclados devido às movimentadas atividades de construção em curso, o que gerou um elevado potencial de mercado e também trouxe um desafio para o fortalecimento do setor de reciclagem. Porém, a viabilidade econômica só seria alcançada se o equipamento instalado na usina não fosse comprado novo $\mathrm{e}$ sim usado, e se fosse atingida a economia de escala na produção dos agregados reciclados. 
Em se tratando do caso brasileiro, o trabalho de Nunes et al. (2007) se mostra como um dos mais importantes estudos da viabilidade de uma implantação de URRCC privada no país. Os autores analisaram duas possibilidades de usinas: uma com capacidade de operação de 20 t.h h $^{-1}$ e outra de 100 t.h $^{-1}$ para municípios brasileiros. Analisando casos de usinas já existentes e considerando as condições do mercado brasileiro na época, eles concluíram que os modelos analisados apenas seriam viáveis economicamente se houvesse algum incentivo do poder público, como subvenção de impostos e taxas ou, dependendo da condição do município, as autoridades públicas poderiam até mesmo assumir a gestão do empreendimento.

Já Costa et al. (2007) fizeram uma análise multivariada dos programas de reciclagem de RCC no Brasil buscando encontrar as variáveis mais relevantes associadas ao sucesso da implementação desses programas nos municípios brasileiros. Os resultados da pesquisa mostraram que existem diferenças marcantes entre as cidades que implantaram programas de reciclagem de RCC e aquelas que não implantaram. Segundo os autores, as variáveis mais significativas encontradas foram: renda média anual do município; existência de programas de coleta seletiva de resíduos sólidos; programas de incentivo para geração de trabalho e renda; e existência de áreas de recepção de entulhos. A maioria das variáveis estava relacionada à gestão municipal.

Diante do exposto, verifica-se que há muitos métodos para se aferir a viabilidade de uma URRCC. Como este trabalho visa a fazer uma análise de investimento em uma usina privada, deve-se determinar, primeiramente, quais os indicadores de viabilidade que serão considerados na análise. Dessa forma, a segunda parte da presente seção apresenta, em síntese, as metodologias que são adotadas nos principais estudos de análise de investimentos.

\section{Análise de Viabilidade Econômica de Projetos de Investimentos}

Existem vários métodos que podem ser utilizados para se fazer uma análise de investimento via Fluxo de Caixa (FC) projetado. Nogas et al. (2011) classificam os métodos de análise de investimentos em três categorias: Método Clássico (MC), Teoria das Opções Reais (TOR) e Metodologia Multi-Índice (MMI). No âmbito da análise de investimentos, o uso do MC e da MMI tem sofrido críticas, uma vez que essas não têm captado o valor da flexibilidade administrativa presente em muitos projetos (RÊGO et al., 2015).

$\mathrm{Na}$ Metodologia Clássica (MC), a Taxa Interna de Retorno (TIR) é vista como proxy de rentabilidade do Projeto de Investimento (PI) a despeito de opinião contrária já encontrada na literatura (MARTIN, 2008; SOUZA \& CLEMENTE, 2008). Na MC, tal como proposto por Grant et al. (1976), valor presente líquido (VPL)>0 e TIR>Taxa Mínima de Atratividade (TMA) são critérios de aceitação para PI. A TOR é similar à $\mathrm{MC}$ no que diz respeito à construção do $\mathrm{FC}$ e respectivo cálculo do VPL. Posteriormente, a TOR insere no FC os efeitos monetários das opções de expandir, contrair, postergar ou abandonar o PI. Ao se adicionar o valor das opções ao VPL da MC, tem-se como resultado

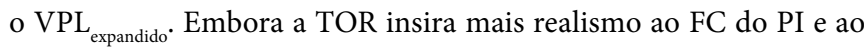
seu processo de gestão, ainda perdura o fato de a taxa de desconto ser composta pela TMA e mais um prêmio pelo risco. O critério de análise para aceitação de um PI é praticamente o mesmo do MC, isto é, $\mathrm{VPL}_{\text {expandido }}>0$ (NOGAS et al., 2011).

Já a MMI se caracteriza pelo uso simultâneo de dois conjuntos de indicadores para representar as dimensões retorno e riscos de certo PI, resultando em informações mais consistentes (SOUZA \& CLEMENTE, 2008; LIMA et al., 2013). O primeiro conjunto é representado por: VPL; VPL Anualizado (VPLA); Índice Benefício/Custo (IBC); Retorno Adicional Proporcionado pela Decisão de Investir (ROIA); e Índice ROIA/TMA. O segundo conjunto, representando os riscos, é constituído por: Índice TMA/TIR - Risco Financeiro ou proxy de P (VPL $\leq 0)$; Índice Payback/N (representando a fração da vida útil necessária para a recuperação do capital investido); Grau de Comprometimento da Receita (risco operacional medido pela fração do faturamento que fica comprometida com o pagamento de custos e despesas); e os índices Riscos de Negócio e Risco de Gestão.

Segundo Harzer (2015), embora muitos dos indicadores de retorno sejam os mesmos utilizados no MC, a significativa contribuição da MMI reside em sintetizar as informações de retorno e de riscos, permitindo o confronto de ambos. Na MMI, o VPL $>0$ ou a TIR $>$ TMA apenas indica que o PI deve continuar sendo analisado. A decisão de recomendar ou não o PI é definida pelo confronto entre o retorno esperado e os riscos percebidos. Essa metodologia força o decisor a um confronto entre as expectativas de retorno e de riscos (SOUZA \& CLEMENTE, 2008; RÊGO et al., 2015).

Recentemente, Lima et al. (2015a) incorporaram à MMI alguns índices para uma análise de sensibilidade, passando a denominá-la de Metodologia Multi-Índice Ampliada (MMIA). A análise de sensibilidade quantifica a vulnerabilidade dos resultados do projeto em relação às mudanças em uma variável, mantendo as demais inalteradas (CORREIA NETO, 2009). Quando uma pequena variação em um parâmetro muda drasticamente a lucratividade de um PI, diz-se que esse é muito sensível ao parâmetro, logo, é importante concentrar esforços para obter menos dados incertos (LIMA et al., 2015a). O propósito da análise de sensibilidade é aprofundar a percepção de risco ao qual o PI é submetido (CASAROTTO \& KOPITTKE, 2010; LIMA et al., 2015a).

Entretanto, um aspecto que deve ser considerado no processo de tomada de decisão é a incerteza. A incerteza introduz uma nova dimensão na análise de um PI. Isso se deve à impossibilidade de controle de futuros eventos com precisão, tais como: o comportamento futuro da economia, vendas futuras, custos com desgaste e manutenção de equipamento. Apesar disso, o tomador de decisão precisa definir, sustentado pelas informações disponíveis, qual a melhor decisão a tomar (SOUZA \& CLEMENTE, 2008; LIMA et al., 2015b). 
Segundo Souza e Clemente (2008), na análise de investimentos, deve-se fazer uso de técnicas que levem em conta a aleatoriedade dos elementos que compõem o FC de um PI. A mais conhecida técnica para lidar com risco e incerteza é o Método de Simulação de Monte Carlo (MSMC), o qual é uma alternativa para a avaliação do risco de um PI (BRUNI et al., 2008). O MSMC é uma técnica que envolve o uso de números pseudoaleatórios e de distribuição de probabilidades para a solução de problemas de inúmeras áreas de pesquisa (GUJARATI, 2002). De acordo com Moore e Weatherford (2005), o MSMC é um dos inúmeros métodos para análise da incerteza. Contudo, sua principal vantagem é determinar quanto uma variação aleatória, que já é conhecida, ou erro afeta a performance da viabilidade do sistema que está sendo modelado. O MSMC pode ser usado para auxiliar na tomada de decisão de investimento, uma vez que os indicadores deixam de ser determinísticos e se tornam estocásticos (LIMA et al., 2015b).

\section{METODOLOGIA}

Para este estudo, foi utilizada uma pesquisa de natureza aplicada com uma abordagem quantitativa, utilizando o método de estudo de caso (CAUCHICK MIGUEL, 2007). De forma a atender o objetivo geral, e considerando os riscos e incertezas envolvidos em um investimento dessa magnitude, optou-se por utilizar a metodologia MMIA neste trabalho, uma vez que ela permite fazer uma avaliação global do PI ao analisar múltiplos indicadores, além de ser mais detalhada nos vários aspectos que a englobam.

Atualmente, a MMIA está sendo implementada em um sistema Web desenvolvido em linguagem PHP, denominado $\$ \Lambda V € \Pi$ - Sistema de Análise da Viabilidade Econômica de Projetos de Investimento ( $\$ \Lambda \mathrm{V} \Pi$, 2015). A MMIA abrange as duas dimensões de retorno e riscos (já apresentadas na MMI) e uma ampliação que envolve a análise de sensibilidade, a qual leva em conta as variações da TMA, dos FCs projetados, dos custos estimados e das receitas esperadas, entre outros. A Tabela 1 mostra as dimensões consideradas com seus respectivos indicadores. As fórmulas de cálculo podem ser encontradas nas referências citadas. Os indicadores de risco de gestão e risco de negócio não foram contemplados neste estudo, pois dependem de análises ambientais mais profundas (HARZER, 2015). O cálculo do tempo de retorno do capital investido (Payback) descontado não será o que é mais frequentemente utilizado: optou-se pelo Payback ajustado, o qual corrige distorções que podem ser geradas no cálculo do Payback de PIs que utilizam-se de financiamentos, principalmente os que apresentam períodos de carência (LIMA et al., 2013).

Ao montar o FC, é preciso quantificar também a depreciação e a inflação, uma vez que elas podem afetar a análise de investimentos. A depreciação pode ser definida como uma despesa relativa à perda de valor de um determinado bem, a qual pode ser acarretada pela deterioração do bem ou pela obsolescência do mesmo. Conforme Instrução Normativa n 162/98 da Receita Federal do Brasil, o prazo de depreciação de máquinas da Construção Civil é de 4 anos, com uma taxa linear de $25 \%$ ao ano. Já para as instalações da Usina, foi considerado o prazo de 10 anos com uma taxa de $10 \%$ ao ano. A inflação representa a perda do poder aquisitivo da moeda, provocada pelo aumento diferenciado do preço de produtos e serviços, que acaba acarretando na diminuição do valor real da moeda (CASAROTTO \& KOPITTKE, 2010; SOUZA \& KLIEMANN NETO, 2012). Este trabalho considerou que todos os elementos do fluxo de caixa são afetados da mesma forma pela inflação. Sendo assim, foi considerado um cenário de inflação homogênea para o período de estudo. Como não há como prever com certeza qual será a inflação do país nos próximos 25 anos, foi considerada a previsão feita pelo Banco Central do Brasil (2015) - o qual realiza uma análise de mercado para os próximos anos - a qual supõe uma estabilização em $4,5 \%$ ao ano.

Para fazer uso de técnicas de análise que levem em conta a aleatoriedade dos elementos que compõem o FC de um PI, recorreu-se ao MSMC. O MSMC busca simular os diversos componentes do FC em

\section{Tabela 1 - Indicadores da Metodologia Multi-Índice Ampliada.}

\begin{tabular}{|c|c|c|c|}
\hline Dimensão & Indicador & Dimensão & Indicador \\
\hline \multirow{10}{*}{$\begin{array}{l}\text { Retorno } \\
\text { (SOUZA \& } \\
\text { CLEMENTE, } \\
\text { 2008; LIMA, } \\
\text { 2016) }\end{array}$} & Valor Presente: VP & \multirow{10}{*}{$\begin{array}{l}\text { Limites de } \\
\text { elasticidade } \\
\text { (LIMA et al., } \\
\text { 2015a) }\end{array}$} & $\Delta \% \mathrm{TMA}$ \\
\hline & $\begin{array}{l}\text { Valor Presente } \\
\text { Líquido: VPL }\end{array}$ & & $\Delta \% F C_{0}$ \\
\hline & $\begin{array}{c}\text { VP da Receita Total: } \\
\text { VP (RT) }\end{array}$ & & $\Delta \% \mathrm{FC}_{\mathrm{j}}$ \\
\hline & $\begin{array}{l}\text { VP do Custo Variável } \\
\text { Total: VP (CVT) }\end{array}$ & & $\Delta \% F C_{0}$ e $F C_{j}$ \\
\hline & $\begin{array}{c}\text { VP do Custo Fixo: } \\
\text { VP(CF) }\end{array}$ & & $\Delta \%$ TMA e FC \\
\hline & $\begin{array}{c}\text { VP do Valor Residual: } \\
\text { VP (VR) }\end{array}$ & & $\Delta \%$ TMA e FC \\
\hline & $\begin{array}{c}\text { Valor Presente } \\
\text { Líquido Anualizado: } \\
\text { VPLA }\end{array}$ & & \multirow{4}{*}{$\Delta \% \mathrm{FC}_{0}$ e $F C_{j}$ e TMA } \\
\hline & $\begin{array}{l}\text { Índice Benefício } \\
\text { Custo: IBC }\end{array}$ & & \\
\hline & $\begin{array}{c}\text { Retorno } \\
\text { Adicional sobre o } \\
\text { Investimento: ROIA }\end{array}$ & & \\
\hline & Índice ROIA/TMA & & \\
\hline \multirow{5}{*}{$\begin{array}{l}\text { Riscos } \\
\text { (SOUZA \& } \\
\text { CLEMENTE, } \\
\text { 2008; LIMA } \\
\text { et al., 2O13) }\end{array}$} & Payback ajustado & & \multirow{5}{*}{ - } \\
\hline & $\begin{array}{l}\text { Taxa Interna de } \\
\text { Retorno: TIR }\end{array}$ & & \\
\hline & Payback ajustado/N & & \\
\hline & Índice TMA/TIR & & \\
\hline & Payback descontado & & \\
\hline
\end{tabular}

$\Delta \%$ : variação percentual; TMA: taxa mínima de atratividade; $\mathrm{FC}_{0}$ : investimento inicial; FC: fluxo de caixa para o período j; Q: quantidade de vendas; PVu: preço de venda unitário; CVu: custo variável unitário; MCu: margem de contribuição unitária;

CF: custo fixo; CT: custo total; RT: receita total; e VR: valor residual.

Fonte: Elaborada a partir de Lima et al. (2015a; 2015b). 
um modelo matemático assim como repetir o processo várias vezes. Dessa forma, pode-se obter a distribuição de probabilidade dos retornos de um PI (GITMAN \& ZUTTER, 2014). Cada componente do FC segue distribuições de probabilidade específicas. Neste trabalho, foram feitas simulações por meio do software Microsoft Excel para as seguintes variáveis: preço de venda dos agregados, custo anual da Usina, geração anual média de RCC e TMA. Para determinação de qual distribuição usar neste trabalho, foram consultados especialistas na área, os quais indicaram que a melhor distribuição a ser usada seria a distribuição triangular, com variação de $20 \%$ para mais e para menos a partir do valor médio estimado para cada variável. A literatura corrobora essa escolha, conforme destacam Harzer (2015), Machado e Ferreira (2012) e Monteiro et al. (2012): em virtude da dificuldade envolvida na identificação das distribuições de probabilidade de cada uma das variáveis mais relevantes, é procedimento usual empregar a distribuição triangular.

Na MSMC, mediante a geração de números aleatórios, valores são obtidos para essas variáveis, resultando em vários FCs e, consequentemente, em vários resultados para cada um dos indicadores considerados para o PI. Pela repetição desse procedimento 10.000 vezes (AGUIAR et al., 2010), pode-se gerar a distribuição de frequências para cada indicador da MMIA, que permite aferir a probabilidade de sucesso ou insucesso do mesmo (HARZER, 2015; MONTEIRO et al., 2012). A próxima etapa foi transformar a demonstração de resultados e os FCs determinísticos em modelos estocásticos com o MSMC.

Em resumo, os principais passos que foram seguidos neste trabalho são:

- dimensionar o PI;

- coletar, organizar e analisar os dados;

- definir a TMA da empresa e o horizonte de planejamento (N) do PI em estudo;

- $\quad$ estimar os custos e receitas anuais e construir a projeção do FC;

- fazer a Simulação de Monte Carlo e incorporá-la ao fluxo de caixa;

- calcular os indicadores de viabilidade econômica e analisá-los: abordagem determinística e estocástica e

- emitir o parecer conclusivo a respeito da viabilidade econômica de implantação do PI.

\section{RESULTADOS E DISCUSSÃO}

Esta seção apresenta os resultados encontrados com a aplicação da metodologia proposta e analisa-os confrontando com a literatura pertinente.

\section{RESULTADOS}

O investimento inicial previsto com base em orçamentos fornecidos por empresas que instalam a URRCC com capacidade produtiva de 10 t.h $^{-1}$ é de R $\$ 451.400,00$. Do montante a ser investido, 75\% pode ser financiado pela Caixa Econômica Federal, cuja taxa de juros é de 9\% ao ano. A amortização pode ser feita em até 15 anos com carência de 5 anos. Para fins deste estudo, considerou-se o Sistema de Amortização Constante (SAC).

Para a análise desse Projeto de Investimento (PI), primeiro projetou-se os valores dos Fluxos de Caixa (FCs) do empreendimento para um horizonte de 25 anos, considerando as receitas e os custos no período em que ocorrerão, de acordo com a inflação estimada e com o aumento da população prevista pelo IBGE. O passo seguinte consistiu em calcular os indicadores determinísticos de retorno e riscos com base na metodologia MMIA. Os resultados obtidos, por meio do sistema Web \$LV€P, estão apresentados na Tabela 2.

Os indicadores de retorno mostram que o VPL, que indica a riqueza proporcionada pelo PI (em toda a sua vida útil), corresponde ao valor esperado de cerca de $\mathrm{R} \$ 967.326,21$, isto é, equivalente a $\mathrm{R} \$ 142.958,50$ por período (VPLA - VPL Anualizado). Nesse PI, a cada unidade monetária investida, estima-se o retorno de 9,5718 unidades, valor observado para o Índice Benefício Custo (IBC). Isso implica em um ROIA de 9,46\% ao período, além da TMA de $14,25 \%$ ao ano, isto é, $66,36 \%$ acima do ganho oportunizado pelo mercado se o capital for aplicado a essa mesma TMA - medido pelo Índice ROIA/TMA. De acordo com esse ganho,

Tabela 2 - Indicadores da Metodologia Multi-Índice Ampliada para o projeto de investimento em estudo.

\begin{tabular}{|c|c|c|c|}
\hline \multicolumn{2}{|l|}{ Retorno } & \multicolumn{2}{|c|}{ Limites de elasticidade } \\
\hline Indicador & Resultado & Indicador & Resultado (\%) \\
\hline VP & $\mathrm{R} \$ 1.080 .176,21$ & $\Delta \% \mathrm{TMA}$ & 490,74 \\
\hline VPL & $\mathrm{R} \$ 967.326,21$ & $\Delta \% \mathrm{FC}_{\mathrm{o}}$ & 857,18 \\
\hline VP (RT) & $\mathrm{R} \$ 5.829 .597,86$ & $\Delta \% F C_{j}$ & 89,55 \\
\hline VP (CVT) & $\mathrm{R} \$ 1.250 .865,14$ & $\Delta \% \mathrm{FC}_{0}$ e FC & 81,08 \\
\hline VP (CF) & $\mathrm{R} \$ 2.223 .676,00$ & $\Delta \%$ TMA e $\mathrm{FC}_{0}$ & 312,07 \\
\hline VP (VR) & $\mathrm{R} \$ 1.578,73$ & $\Delta \%$ TMA e FC & 75,73 \\
\hline VPLA & $\mathrm{R} \$ 142.958,50$ & $\begin{array}{c}\Delta \% \mathrm{FC}_{0} \text { e } F C_{j} \mathrm{e} \\
\text { TMA }\end{array}$ & 69,58 \\
\hline IBC & $\mathrm{R} \$ 9,57$ & - & - \\
\hline ROIA & $9,46 \%$ & - & - \\
\hline Índice ROIA/TMA & $66,36 \%$ & - & - \\
\hline \multicolumn{4}{|l|}{ Riscos } \\
\hline Indicador & Resultado & - & - \\
\hline Payback ajustado & 9 anos & - & - \\
\hline TIR & $84,18 \%$ & - & - \\
\hline $\begin{array}{l}\text { Payback } \\
\text { ajustado/N }\end{array}$ & $36,00 \%$ & - & - \\
\hline Índice TMA/TIR & $16,93 \%$ & - & - \\
\hline
\end{tabular}

$\Delta \%$ : variação percentual; TMA: taxa mínima de atratividade; $\mathrm{FC}_{0}$ : investimento inicial; FC: fluxo de caixa para o período j; Q: quantidade de vendas; PVu: preço de venda unitário; CVu: custo variável unitário; MCu: margem de contribuição unitária; CF: custo fixo; CT: custo total; RT: receita total; e VR: valor residual. 
o PI pode ser classificado na categoria de retorno de grau médio-alto, seguindo a classificação proposta por Lima (2016).

Os indicadores de riscos mostram que, na relação entre o Payback e a vida útil considerada $(\mathrm{N})$, medida pelo índice Payback/N, o PI apresenta risco de $36,00 \%$, indicando um grau baixo-médio (LIMA, 2016) de o projeto não se pagar (SOUZA \& CLEMENTE, 2008). Ao medir a proximidade entre a TMA e a TIR, utilizando o Índice TMA/TIR, o PI também apresentou um indicativo de grau de risco classificado como baixo (16,93\%). Quanto ao indicador Payback, como foi explicado na metodologia, aqui optou-se por utilizar o Payback ajustado (LIMA et al., 2013), o qual objetiva corrigir possíveis distorções que possam ser geradas no cálculo do Payback. Dessa forma, o índice Payback ajustado mostrou que o PI em estudo levará 9 anos para recuperar o capital investido.

No tocante aos limites de elasticidade, o PI em estudo torna-se inviável economicamente, se a TMA aumentar mais do que 490,74\% ou se o investimento inicial $\left(\mathrm{FC}_{0}\right)$ realizado aumentar mais do que $857,18 \%$ do valor projetado, desde que sejam mantidos constantes os demais parâmetros. Já a variação entre o Investimento inicial, demais fluxos de caixa e a TMA não pode ser maior que $69,58 \%$, para que o PI continue sendo viável.

A próxima etapa foi transformar as projeções dos fluxos de caixa determinísticos em modelos estocásticos com o MSMC. Para a composição do modelo estocástico, foi utilizada a distribuição triangular, com variabilidade de $20 \%$ para mais e para menos a partir dos dados coletados, seguindo orientação de especialistas da área consultados. A simulação de Monte Carlo foi elaborada abrangendo 10.000 interações para cada variável aleatória. A partir dessas simulações, geraram-se os novos FC para os 25 períodos e, com eles, foram estimados os novos parâmetros de riscos, retorno e de sensibilidade.

No MSMC, o VPL médio ficou em aproximadamente R \$ 899.350,92, menor que na abordagem determinística. O IBC também se mostrou mais moderado, com 2,99 unidades de retorno para cada unidade investida. Na média, o Índice ROIA/TMA se estabeleceu em 32,62\%, sendo considerado de risco baixo-médio (LIMA, 2016). No que diz respeito às probabilidades do VPL, verificou-se que, com 10.000 simulações, a probabilidade de o VPL ser menor que 0 (zero) é de apenas $0,22 \%$. Por outro lado, a probabilidade de o PI ser menor que R\$1.000.000,00 é de $62,49 \%$ e a probabilidade de ele ser maior que $\mathrm{R} \$ 500.000,00$ é de $89,68 \%$. A Figura 1 mostra a distribuição de probabilidades do VPL geradas a partir dessas simulações.

\section{DISCUSSÃO}

No aspecto financeiro, a implantação da URRCC mostrou-se economicamente viável. A partir dos resultados gerados pelo sistema Web \$LV€P, verificou-se que todos os indicadores foram positivos. Os indicadores da dimensão "Retorno" mostraram que o PI em estudo apresenta boa perspectiva de retorno, com destaque para o VPLA, que mostrou um rendimento anual satisfatório para o investidor, e o indicador ROIA/ TMA que apresentou um retorno médio-alto do PI (66,36\%). Além disso, os indicadores da dimensão "Riscos" também se mostraram favoráveis. Mesmo utilizando o índice Payback ajustado, espera-se que o retorno do capital investido ocorra em 9 anos. Os índices Payback/N e TMA/ TIR ficariam em $36,00 \%$ e $16,93 \%$, respectivamente, podendo ser categorizados como índices de grau de riscos baixo-médio (LIMA, 2016).

No tocante aos limites de elasticidade, verificou-se que o PI em estudo é mais sensível às reduções nos Fluxos de Caixa (FCs). Os indicadores de sensibilidade, considerando a escala proposta por Lima (2016), mostram que a TMA deveria variar na ordem de 490,75\% para que o mercado financeiro propiciasse o mesmo retorno do PI, ou seja, a sensibilidade se mostra de grau excepcional, mesmo grau apresentado pela $\Delta \%$ Custos de implantação $\left(\mathrm{FC}_{0}\right)$ de $857,18 \%$ e pela $\Delta \%$ TMA e $\mathrm{FC}_{0}(312,07 \%)$. A $\Delta \% \mathrm{FC}_{\mathrm{j}}$ de $89,55 \%$ e a $\Delta \%$ entre $\mathrm{FC}_{0}$ e FC de $81,08 \%$ se mostraram sensíveis de grau alto. Por fim, a $\Delta \% \mathrm{TMA}$ e $\mathrm{FCj}$ e a $\Delta \% \mathrm{FC}_{0}, \mathrm{FC}_{\mathrm{j}}$ e TMA se mostraram de grau médio-alto. Diante disso, a análise preliminar mostra que a decisão de investir no PI em estudo se apresenta como uma boa opção de investimento.

Entretanto, devido à aleatoriedade dos componentes do FC projetado, recorreu-se à Simulação de Monte Carlo como forma de melhor avaliar o risco envolvido no PI. Por meio dessa técnica, verificou-se que o VPL médio encontrado, utilizando-se de 10.000 simulações, é menor do que o calculado na abordagem determinística, bem como o VPLA, o IBC, o ROIA e o ROIA/TMA, ou seja, o retorno esperado se mostrou menor do que o apontado pelo enfoque estático, porém, ainda positivo. Já os índices de riscos resultaram em valores menores na abordagem estocástica, ou seja, há um risco menor envolvido no projeto do que o estimado pela abordagem determinística, mostrando a importância dessa abordagem.

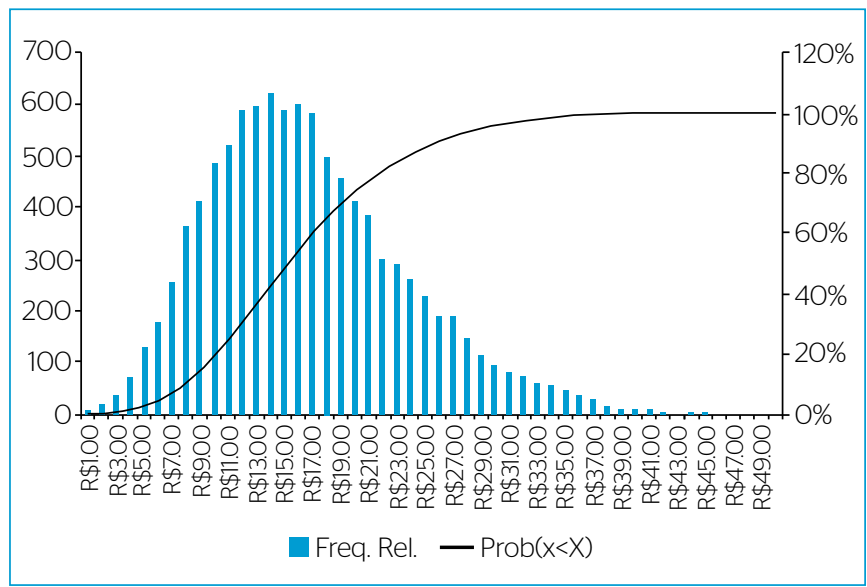

Figura 1 - Distribuição de probabilidades do valor presente líquido. 


\section{CONSIDERAÇÕES FINAIS}

O setor da construção civil é expressivo para o crescimento do país. Esse setor é responsável pela contratação de parte da mão de obra bem como tem expressiva contribuição para o Produto Interno Bruto (PIB) brasileiro. Porém, na contramão desses benefícios, ele é um grande gerador de resíduos, os quais geralmente são destinados incorretamente. Uma das formas de destinação correta desses resíduos seria por meio de usinas de reciclagem de resíduos da construção civil e demolição. Diante disso, este artigo teve como objetivo verificar a viabilidade econômico-financeira da implantação de uma URRCC no município de Pato Branco (PR).

Os resultados encontrados satisfazem os objetivos propostos. A análise determinística apresentou resultados favoráveis à decisão de investir no projeto. Por outro lado, a análise estocástica apresentou resultados mais contidos. Ainda assim, positivos, mostrando que há alta probabilidade $(99,78 \%)$ de que o investimento em uma
URRCC seja economicamente viável. Entretanto, os índices de elasticidade mostraram que é preciso monitorar as receitas esperadas e os custos estimados com mais atenção, ou seja, será preciso um trabalho, por parte dos gestores da URRCC, em prol de sempre estarem buscando aumentar a demanda pelos agregados reciclados, como maneira de aumentar a receita da usina, desde que a mesma não ultrapasse sua capacidade de produção.

Como sugestão para estudos futuros, propõe-se que se faça um estudo de mercado mais aprofundado a respeito dos custos e receitas de instalação da URRCC, bem como dos custos de manutenção e funcionamento da mesma. Também se sugere que sejam feitos mais testes estatísticos sobre as distribuições de probabilidades mais adequadas para cada uma das principais variáveis aleatórias intervenientes no desempenho econômico do PI em estudo, para se obter a maior segurança possível nos resultados. Por fim, sugere-se a incorporação da Teoria da Opções Reais (TOR) para contemplar algumas flexibilidades gerenciais.

\section{REFERÊNCIAS}

AGUIAR, G.; ALVES, C.C.; HENNING, E. (2010) Gerenciamento de Projetos: Simulação de Monte Carlo via a Ferramenta Simular. In: XXX Encontro Nacional de Engenharia de Produção, Anais... São Carlos, p. 1-13.

ASSOCIAÇÃO BRASILEIRA DE EMPRESAS DE LIMPEZA PÚBLICA E RESIDUOS ESPECIAIS - ABRELPE. (2014) Panorama nos resíduos sólidos no Brasil: São Paulo, 2014.

ASSOCIAÇÃO BRASILEIRA DE NORMAS TÉCNICAS - ABNT. (2004) NBR 15114: Resíduos sólidos da construção civil - Áreas de reciclagem Diretrizes para projeto, implantação e operação, Rio de Janeiro.

ASSOCIAÇÃO BRASILEIRA PARA RECICLAGEM DE RESÍDUOS DA CONSTRUÇÃO CIVIL E DEMOLIÇÃO - ABRECON. (2014) Relatório: pesquisa setorial 2014/2015, São Paulo, SP.

AZEVEDO, G.O.D.D., KIPERSTOK, A., MORAES, L.R.S. (2006) Resíduos da construção civil em Salvador: os caminhos para uma gestão sustentável. Engenharia Sanitária e Ambiental, v. 11, n. 1, p. 65-72.

BANCO CENTRAL DO BRASIL - BCB. (2015) Relatório de Inflação, v. 17, n. 3, p. 1-125

BRASIL. (2002) Ministério do Meio Ambiente - Conselho Nacional do Meio Ambiente - CONAMA. Resolução no. 307, de 05 de julho de 2002. Brasília. Publicada no DOU no 136, de 17/07/2002, p. 95-96. Disponível em: <http://www.mma.gov.br>. Acesso em: 15/08/2015.

BRUNI, A.L.; FAMÁ, R.; SIQUEIRA, J.O. (1998) Análise do risco na avaliação de projetos de investimento: uma aplicação do método de Monte Carlo. Cadernos de Pesquisa em Administração, v.1, n. 6, p. 62-74.

CASAROTTO, N.F. \& KOPITTKE, B.H. (2O10) Análise de Investimentos Matemática Financeira, Engenharia Econômica, Tomada de Decisão, Estratégia Empresarial. 11 ed. São Paulo: Atlas, 411p.

CAUCHICK MIGUEL, P.A. (2007) Estudo de caso na engenharia de produção: estruturação e recomendações para sua condução. Produção, v. 17, n. 1, p. 216-229.

COELHO, A. \& BRITO, J. (2013) Economic viability analysis of a construction and demolition waste recycling plant in Portugal-part I: location, materials, technology and economic analysis. Journal of Cleaner Production, v. 39, p. 338-52.

CORREIA NETO, J.F. (2009) Elaboração e avaliação de projetos de investimento: considerando o risco. Rio de Janeiro: Elsevier, 266 p.

COSTA, N.; COSTA JR, N.; LUNA, M.; SELIG, P.; ROCHA, J. (2007) Planejamento de programas de reciclagem de resíduos de construção e demolição no Brasil: uma análise multivariada. Engenharia Sanitária e Ambiental, v. 12, n. 4, p. 446-56.

DURAN, X.; LENIHAN, H.; O' REGAN, B. (2006) A model for assessing the economic viability of construction and demolition waste recycling and the case of Ireland. Resources, Conservation and Recycling, v. 46, n. 3, p. 302-20.

GITMAN, L.J. \& ZUTTER, C.J. (2014) Principles of Managerial Finance. 14. ed. Ed. Prentice Hall, 944p. 
GRANT, E.L.; IRESON, W.G.; LEAVENWORTH. R.S. (1976) Principles of Engineering Economy, 6a Ed. Nova York: Ronald Press.

GUJARATI, D.N. (2002) Econometria básica. 3 ed. São Paulo: Makron Books, 783p.

HARZER, J.H. (2015) Indicadores de riscos em projetos de investimentos: uma contribuição à Metodologia Multi-índice. 2015. Tese (Doutorado em Administração) Pontifícia Universidade Católica do Paraná, Curitiba.

KARTAM, N.; AL-MUTAIRI, N:; AL-GHUSAIN, I:; AL-HUMOUD, J. (2004) Environmental management of construction and demolition waste in Kuwait. Waste Management, v. 24, n. 10, p. 1049-59.

LIMA, J.D. de; SCHEITT, L.C.; BOSCHI, T.F; SILVA, N.J.; MEIRA, A.A.; DIAS, G.H. (2013) Propostas de ajuste no cálculo do payback de projetos de investimentos financiados. Custos e @gronegócio on line, v. 9, n. 4, p. 162-80

LIMA, J.D.; TRENTIN, M.G.; OLIVEIRA, G.A.; BATISTUS, D.R.; SETTI, D. (2015a) A systematic approach for the analysis of the economic viability of investment projects. International Journal of Engineering Management and Economics, v. 5, n. 1/2, p. 19-34.

LIMA, J.D.; TRENTIN, M.G.; OLIVEIRA, G.A.; BATISTUS, D.R.; SETTI, D. (2015b) Systematic analysis of economic viability with stochastic approach: a proposal for investment. In: XXI International Conference on Industrial Engineering and Operations Management, Anais... Aveiro, Portugal, p. 1-8.

LIMA, J.D. (2016) Manual de Análise da Viabilidade Econômica de Projetos de Investimentos (MAVEPI): abordagem determinística. Universidade Tecnológica Federal do Paraná (UTFPR - Câmpus Pato Branco). Programa de Pós-graduação em Engenharia de Produção e Sistemas (PPGEPS). 2016. Disponível em: <http://pb.utfpr.edu.br/savepi/cadastrar.php>. Acesso em: fev. 2016.

MACHADO, N.R.S. \& FERREIRA, A.O. (2012) Método de simulação de Monte Carlo em planilha Excel: desenvolvimento de uma ferramenta versátil para análise quantitativa de riscos em gestão de projetos. Revista de Ciências Gerenciais, v. 16, n. 23, p. 223-44.

MIRANDA, L.F.R.; ÂNGULO, S.C.; CARELI, E.D. (2009) A reciclagem de resíduos de construção e demolição no Brasil: 1986-2008. Ambiente Construido, v. 9, n. 1, p. 57-71.

MONTEIRO, C.A; SANTOS, L.S; WERNER, L. (2O12) Simulação de Monte Carlo em decisão de investimento para implantação de projeto hospitalar. In: XXXII Encontro Nacional de Engenharia de Produção, Anais... Bento Gonçalves, Rio Grande do Sul.
MOORE, J.H.; WEATHERFORD, L.R. (2005) Tomada de decisão em administração com planilhas eletrônicas. Porto Alegre: Bookman.

NOGAS, P.S.M.; SOUZA, A.; SILVA, W.V. (2011) Análise de investimentos: uma contribuição probabilística ao índice TMA/TIR da Metodologia Multi-índice. Revista Iberoamericana de Ciencias Empresariales y Economía, v. 2, n. 2, p. 43-55.

NUNES, K.R.A. (2004) Avaliação de investimentos e de desempenho de centrais de reciclagem para resíduos sólidos de construção e demolição. 2004 Tese (Doutorado) Departamento de Engenharia de Produção, Universidade Federal do Rio de Janeiro, Rio de Janeiro.

NUNES, K.R.A; MAHLER, C.F; VALLE, R; NEVES, C. (2007) Evaluation of investments in recycling centers for construction and demolition wastes in Brazilian municipalities. Waste Management, v. 27, n. 11, p. 1531-40.

PINTO, T.P. (1999) Metodologia para a gestão diferenciada de Resíduos sólidos da construção urbana. 1999. Tese (Doutorado) Escola Politécnica da Universidade de São Paulo. Departamento de Engenharia de Construção Civil, São Paulo.

RÊGO, T.F.; CORREIA, A.M.M.; ARAÚJO, J.A.R.; GURGEL, J.C: SOUZA, A. (2015) Expectativas de retorno e riscos percebidos no agronegócio corante natural de caju para o mercado B2B. Custos e Agronegócio on-line, v. 11, n. 2, p. 370-400.

SANTOS, F.R. \& POMPEU, R.B. (2014) Logística reversa de resíduos da construção civil: análise de viabilidade econômica. Revista Tecnológica Fatec Americana, v. 2, n. 1, p. 105-20.

SCREMIN, E.B.; CASTILHOS JR., A.B.; ROCHA, J.C. (2014) Sistema de apoio ao gerenciamento de resíduos de construção e demolição para municípios de pequeno porte. Engenharia Sanitária e ambiental, v. 19, n. 2, p. 203-06.

SOUZA, A. \& CLEMENTE, A. (2008) Decisões Financeiras e Análise de Investimentos: Fundamentos, técnicas e aplicações. 6 ed. São Paulo: Atlas.

SOUZA, J.S. \& KLIEMANN NETO, F.J. (2012) O impacto da incorporação da inflação na análise de projetos de investimentos. Produção, v. 22, n. 4, p. 709-17.

$\$ \Lambda \bigvee € \Pi$. Sistema de Análise da Viabilidade Econômica de Projetos de Investimento, 2015. Disponível em: < http://pb.utfpr.edu.br/ savepi/cadastrar.php>. Acesso em: fev. 2016.

ZHAO, W.; LEEFTINK, R.B.; ROTTER, V.S. (2010) Evaluation of the economic feasibility for the recycling of construction and demolition waste in China: the case of Chongqing. Resources, Conservation and Recycling, v. 54, n. 6, p. 377-89. 